\title{
Isolation and characterization of nanocrystalline cellulose from sugar palm fibres (Arenga Pinnata)
}

\begin{abstract}
ABSTRCT
Cellulose was extracted from sugar palm fibres (Arenga pinnata) by conducting delignification and mercerization treatments. Subsequently, sugar palm nanocrystalline celluloses (SPNCCs) were isolated from the extracted cellulose with $60 \mathrm{wt} \%$ concentrated sulphuric acid. The chemical composition of sugar palm fibres were determined at different stages of treatment. Structural analysis was carried out by Brunauer-Emmett-Teller (BET), X-ray diffraction (XRD) and Fourier transform infrared spectroscopy (FT-IR). Morphological analysis of extracted cellulose and isolated nanocrystalline cellulose (NCCs) was investigated by using field emission scanning electron microscopy (FESEM), atomic force microscopy (AFM), and transmission electron microscopy (TEM). The thermal stability of sugar palm fibres at different stages of treatment was investigated by thermogravimetric analysis (TGA). The results showed that lignin and hemicellulose were removed from the extracted cellulose through the delignification and mercerization process, respectively. The isolated SPNCCs were found to have length and diameters of $130 \pm 30 \mathrm{~nm}$ and $9 \pm 1.96 \mathrm{~nm}$, respectively.
\end{abstract}

Keyword: Sugar palm fibres; Cellulose; Sugar palm nanocrystalline cellulose; Nanofibre; Thermal behavior; Acid hydrolysis. 